INPLASY

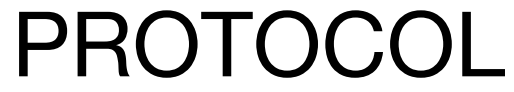

To cite: García-Hermoso et al. Associations between Mediterranean Diet and healthy habits among youths. Inplasy protocol 202040032. doi:

10.37766/inplasy2020.4.0032

Received: 06 April 2020

Published: 06 April 2020

Corresponding author: Antonio García-Hermoso

antonio.garciah@unavarra.es

Author Affiliation:

Navarrabiomed, Complejo

Hospitalario de Navarra

Support: None

Review Stage at time of this submission: Preliminary searches.

Conflicts of interest: None.

\section{Associations between Mediterranean Diet and healthy habits among youths}

García-Hermoso, A; Ezzatvar, Y2; López-Gil JF3; Izquierdo M4; Ramírez-Vélez R ${ }^{5}$.

Review question / Objective: The aim of this study is to metaanalyze the associations between Mediterranean Diet and physical activity, sedentary behavior and physical fitness among children and adolescents aged 3-18 years old.

Rationale: The relationship between adherence to the Mediterranean Diet and physical activity, sedentary behaviors and physical fitness level has been analyzed in several studies.

Condition being studied: Apparently healthy youth aged 3-18 years old.

Eligibility criteria: To be eligible for inclusion in the metaanalysis, studies needed to meet the following criteria: (i) exposure: Mediterranean Diet measured using the KIDMED test or similar; (ii) participants: generally healthy population aged 3 up to 18 years (mean age); and (iii) outcomes analyzed: PA, screen time and physical fitness; (iv) study design: crosssectional and prospective cohort studies.

INPLASY registration number: This protocol was registered with the International Platform of Registered Systematic Review and Meta-Analysis Protocols (INPLASY) on 06 April 2020 and was last updated on 06 April 2020 (registration number INPLASY202040032).

\section{INTRODUCTION}

Review question / Objective: The aim of this study is to meta-analyze the associations between Mediterranean Diet and physical activity, sedentary behavior and physical fitness among children and adolescents aged 3-18 years old.

Condition being studied: Apparently healthy youth aged 3-18 years old. 
Rationale: The relationship between adherence to the Mediterranean Diet and physical activity, sedentary behaviors and physical fitness level has been analyzed in several studies.

\section{METHODS}

Search strategy: We will used variations of the term and Mediterranean Diet (e.g., Mediterranean, diet, adherence), children and adolescents (e.g., child, children, adolescent, adolescents, youth), physical activity (e.g., active, exercise, physical inactivity), sedentary behaviors (e.g., sitting time, screen time, screen media), and physical fitness (e.g., cardiorespiratory fitness, aerobic fitness, muscular strength).

Participant or population: Generally healthy population aged 3 up to 18 years (mean age).

\section{Intervention: None.}

Comparator: None.

Study designs to be included: Crosssectional and prospective cohort studies.

Eligibility criteria: To be eligible for inclusion in the meta-analysis, studies needed to meet the following criteria: (i) exposure: Mediterranean Diet measured using the KIDMED test or similar; (ii) participants: generally healthy population aged 3 up to 18 years (mean age); and (iii) outcomes analyzed: PA, screen time and physical fitness; (iv) study design: crosssectional and prospective cohort studies.

Information sources: We will systematically searched MEDLINE, EMBASE and SPORTDiscus electronic databases for articles (two independent authors). Searching was restricted to published articles in the English and Spanish languages.

Main outcome(s): - Physical activity: validated questionnaires or other tools (e.g., accelerometer). - Sedentary behavior: sitting time, screen time, screen media, electronic media, internet use, computer use, mobile phone use, television watching, video game. - Physical fitness: cardiorespiratory fitness, muscular fitness and speed-agility.

Quality assessment / Risk of bias analysis: The Quality Assessment Tool for Observational Cohort and Cross-sectional Studies will be used to evaluate the risk of bias. The checklist comprise 14 items for longitudinal research, of which only 11 could be applied to cross-sectional studies. Each item of methodological quality will be classified as "yes", "no" or "not reported".

Strategy of data synthesis: Researchers will create tables to summarize the included studies and show their key characteristics and any important questions related to the aim of this review. Reviewers will determine whether a metaanalysis is possible, once data has been extracted. If it is possible to carry out a meta-analysis, Comprehensive MetaAnalysis software will be used to combine the extracted data using an inverse variance model. We will compare adjusted and unadjusted estimates separately for each outcome. A fixed-effect model will be used if there is no evidence of heterogeneity, otherwise a random-effect model will be used.

Subgroup analysis: We will identified potential moderator variables a priori. The variables wiil be sex and age (children $<12$ years and adolescents $\geq 12$ years of mean age), by stratifying meta-analyses by each of these factors.

Sensibility analysis: Sensitivity analysis will be performed to determine whether any single study with extreme findings had an undue influence on the overall results.

Language: English

Country(ies) involved: Spain.

Keywords: Mediterranean Diet; Physical activity; Sedentary behavior; Physical fitness; Youth. 